\title{
On the Coupling of Wind Stress and Sea Surface Temperature
}

\author{
R. M. Samelson, E. D. Skyllingstad, D. B. Chelton, S. K. Esbensen, L. W. O’Neill, and N. Thum \\ College of Oceanic and Atmospheric Sciences, Oregon State University, Corvallis, Oregon
}

(Manuscript received 25 January 2005, in final form 5 August 2005)

\begin{abstract}
A simple quasi-equilibrium analytical model is used to explore hypotheses related to observed spatial correlations between sea surface temperatures and wind stress on horizontal scales of $50-500 \mathrm{~km}$. It is argued that a plausible contributor to the observed correlations is the approximate linear relationship between the surface wind stress and stress boundary layer depth under conditions in which the stress boundary layer has come into approximate equilibrium with steady free-atmospheric forcing. Warmer sea surface temperature is associated with deeper boundary layers and stronger wind stress, while colder temperature is associated with shallower boundary layers and weaker wind stress. Two interpretations of a previous hypothesis involving the downward mixing of horizontal momentum are discussed, and it is argued that neither is appropriate for the warm-to-cold transition or quasi-equilibrium conditions, while one may be appropriate for the cold-to-warm transition. Solutions of a turbulent large-eddy simulation numerical model illustrate some of the processes represented in the analytical model. A dimensionless ratio $\gamma_{\tau A}$ is introduced to measure the relative influence of lateral momentum advection and local surface stress on the boundary layer wind profile. It is argued that when $\gamma_{\tau A}<1$, and under conditions in which the thermodynamically induced lateral pressure gradients are small, the boundary layer depth effect will dominate lateral advection and control the surface stress.
\end{abstract}

\section{Introduction}

Satellite observations of sea surface temperature (SST) and wind stress have recently revealed systematic correlations between warm SST anomalies and enhanced wind stress on horizontal scales of $50-500 \mathrm{~km}$ in both the Tropics and the extratropics (e.g., Liu et al. 2000; Chelton et al. 2001; Hashizume et al. 2001; Polito et al. 2001; O'Neill et al. 2003; see also the reviews in Chelton et al. 2004; Xie 2004). One possible mechanism that has been invoked in several studies (e.g., Sweet et al. 1981; Wallace et al. 1989; de Szoeke and Bretherton 2004) as a possible source of these correlations involves changes in the stability of the boundary layer, which are presumed to control the "downward mixing" of momentum from a low-level jet to the lower boundary layer. In this scenario, it is argued that the enhanced winds over warm water are maintained by enhanced downward fluxes, induced by convective instability, of

Corresponding author address: R. M. Samelson, College of Oceanic and Atmospheric Sciences, Oregon State University, 104 COAS Admin. Bldg., Corvallis, OR 97331-5503.

E-mail: rsamelson@coas.oregonstate.edu horizontal momentum from the faster flow above to the slower near-surface flow. Other possible mechanisms include flows driven by thermodynamically induced pressure gradients (e.g., Lindzen and Nigam 1987; Wai and Stage 1989; Small et al. 2003).

Here, a new hypothesis for this observed correlation is advanced for the case of quasi-equilibrium conditions, and the viability of the downward-mixing mechanism is reconsidered. A simple analytical model of the marine atmospheric boundary layer is used to illustrate the concepts. The main goal is to clarify the effect of variations in the intensity of turbulent mixing within the boundary layer on near-surface winds under quasiequilibrium conditions by considering a model that retains a basic representation of the essential physics, but is simple enough to yield explicit analytical solutions whose dependence on parameters can be readily identified and interpreted. The discussion of numerical model results by Wai and Stage (1989) anticipates some aspects of the present hypothesis, but focuses on the pressure gradient mechanism and on variability with smaller horizontal scales. The present study does not address the pressure gradient mechanism or the force balances in the complex transition region in the immediate vicinity of SST fronts. 


\section{Quasi-equilibrium models}

\section{a. Nonrotating integral momentum balance}

Consider the downstream momentum balance in a turbulent marine atmospheric boundary layer with flow in, say, the meridional direction. Define $z=h$, the top of the boundary layer, as the level at which the (kinematic) turbulent stress $\tau=-\left\langle v^{\prime} w^{\prime}\right\rangle$ vanishes, where $v^{\prime}$ and $w^{\prime}$, respectively, are meridional and vertical fluctuating turbulent velocities. The depth $h$ might be estimated from physical balances (e.g., by MoninObukhov theory in the stable case), but for the present purposes it is adequate to specify it directly. Assume a fixed meridional pressure gradient $G=\rho_{0}^{-1} p_{y}$, where $\rho_{0}$ is a constant reference density, and (for simplicity; note also that this follows a standard assumption in fluid boundary layer theory) take $G$ to be independent of depth in the boundary layer. Neglect rotation, time dependence, and horizontal variations, and consider only the vertical divergence of the vertical turbulent stress. Numerical solutions discussed below (section 4c) address the general quasi-equilibrium case, with rotation, stratification, nonlinearity, and time dependence. As discussed below, those solutions support the qualitative picture illustrated here through the consideration of simple analytical models.

With these assumptions, the meridional momentum equation in the boundary layer reduces to a balance between pressure gradient and stress divergence,

$$
0=-G+d \tau / d z \text {. }
$$

Integrate vertically over the boundary layer and divide by the boundary layer depth $h$ to obtain the vertically averaged momentum balance

$$
0=-G-\tau_{s} / h,
$$

where $\tau_{s}=\tau(z=0)$ is the surface stress. The balance (2.2) is between the pressure gradient force and the mean vertical divergence of the vertical turbulent stress. From this relation, it is immediately evident that, under these assumptions, the surface stress $\tau_{s}$ is independent of the internal profile of the turbulent stress, that is, of the form of $\tau(z)$ for $z>0$. For fixed pressure gradient force $G$, however, $\tau_{s}$ increases linearly with the boundary layer depth $h$, because the stress vanishes by assumption at the top of the stress boundary layer, and the stress divergence is distributed over a deeper layer.

\section{b. Rotational effects}

When rotation is included, an integral relation of the form (2.2) may still be derived for the downwind momentum balance, where the downwind direction is defined in terms of the vertically integrated flow. How-

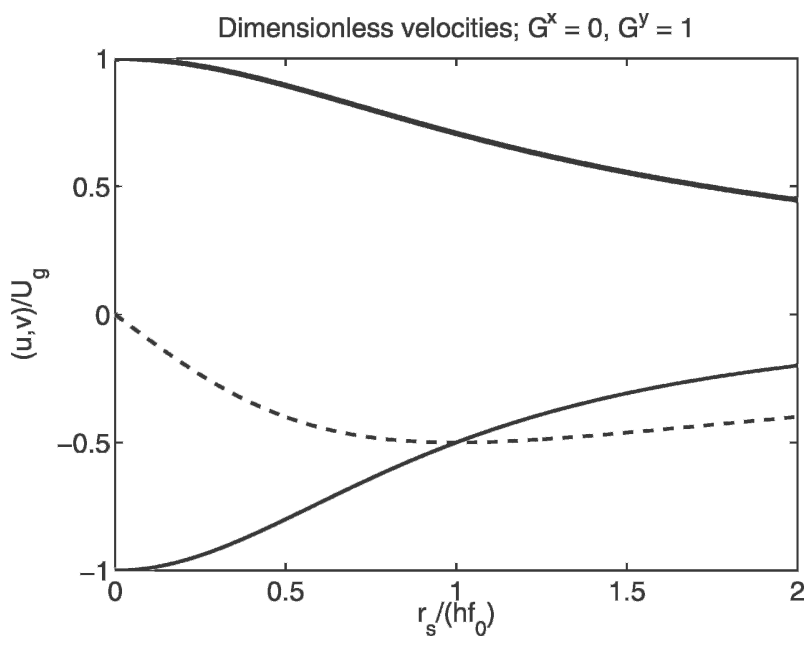

FIG. 1. Dimensionless magnitude $U / U_{g}$ (thick solid line) and zonal (solid) and meridional (dashed) components of horizontal wind in the slab boundary layer model vs dimensionless ratio $r_{s} /(h f)$ of rotational to frictional time scales. The dimensionless pressure gradient is scaled to one and oriented meridionally $(G=$ 1 ). For fixed pressure gradient $G$, drag coefficient $r_{s}$, and Coriolis parameter $f$, wind speed and stress $r_{s} U$ increase with increasing boundary layer depth $h$.

ever, because this direction depends on the wind and turbulent stress profiles as well as the pressure gradient, these profiles must be determined before a similar dependence of surface stress on boundary layer depth can be inferred.

A standard, analytically accessible boundary layer model that provides these profiles and illustrates this dependence in the presence of rotation is a slab mixedlayer model, in which the horizontal velocities are assumed independent of height in the boundary layer. With a linear surface stress parameterization, the corresponding momentum equations are

$$
\begin{aligned}
-f v & =-r_{s} u / h, \\
f u & =-G-r_{s} v / h .
\end{aligned}
$$

Here $f$ is the Coriolis parameter, $G$ and $h$ are as before, and $r_{s}$ is a linearized surface drag coefficient. The solution is

$$
\mathbf{u}=(u, v)=\left(f^{2}+r_{s}^{2} / h^{2}\right)^{-1} G\left(-f,-r_{s} / h\right),
$$

with wind speed magnitude

$$
U=\left(u^{2}+v^{2}\right)^{1 / 2}=\left(f^{2}+r_{s}^{2} / h^{2}\right)^{-1 / 2} G .
$$

For fixed $f, r_{s}$, and $G$, the magnitudes $U$ and $r_{s} U$ of the wind speed and the surface stress, respectively, both increase monotonically with $h$ toward the geostrophic limit (Fig. 1). If $h$ changes so that the ratio of the rotational time scale $1 / f$ to the frictional time scale $h / r_{s}$ varies between 0 and 2, the surface stress changes by a 
factor of 2. Thus, in this model, the presence of rotation does not qualitatively alter the strong dependence of surface stress on boundary layer depth as suggested by the nonrotating balance (2.2).

A second, standard boundary layer model that includes rotation is the classical Ekman layer (e.g., Pedlosky 1987), for which a similar proportionality between stress and boundary layer depth also arises for fixed pressure gradient. In that case, the surface stress is

$$
\tau_{s}=\delta G\left(\cos \theta_{s}, \sin \theta_{s}\right)
$$

where $\delta$ is an Ekman depth, proportional to the square root of the eddy diffusivity, and $\theta_{s}=\theta_{G} \pm \pi / 4$ is the hemispherically dependent direction of the surface stress, with $G$ now the magnitude and $\theta_{G}$ the direction of the pressure gradient. The angular difference $\pm \pi / 4$ between pressure gradient and surface stress is the Ekman veering resulting from frictional effects in the boundary layer. Thus, for fixed $G$ and $\theta_{G}$ in (2.7), the surface stress $\tau_{s}$ is proportional to the Ekman depth $\delta$. However, in the Ekman-layer theory, it is not possible to separate the internal mixing processes from the surface stress parameterization, because both are proportional to the same eddy diffusivity.

\section{c. A two-layer model: Internal mixing}

Consider now a simple representation of the vertical structure of the boundary layer, in which the boundary layer is divided into two sublayers of equal fixed thickness $h / 2$, each of which is a slab layer with vertically uniform horizontal velocity $\mathbf{u}_{j}$, where $j=1,2$ (Fig. 2). This division provides an intermediate level at which the intensity of the internal turbulent mixing within the boundary layer can be conveniently prescribed in a manner that is independent of the surface stress parameterization. Let layer 1 represent the lower half of the boundary layer, and layer 2 the upper half. The momentum equations are

$$
\begin{aligned}
-f v_{1} & =2\left(\tau_{i}^{x}-\tau_{s}^{x}\right) / h, \\
f u_{1} & =-G+2\left(\tau_{i}^{y}-\tau_{s}^{y}\right) / h, \\
-f v_{2} & =-2 \tau_{i}^{x} / h, \quad \text { and } \\
f u_{2} & =-G-2 \tau_{i}^{y} / h,
\end{aligned}
$$

where the entrainment stress at the top of the boundary layer is assumed to vanish, $\tau_{i}$ is the interfacial stress, and as before $\tau_{s}$ is the surface stress.

This simple model of the vertical structure may be closed by specifying the dependence of the turbulent stresses on the flow variables. For simplicity, assume linear drag laws for the interfacial and surface stress vectors,

$$
\tau_{i}=r_{i}\left(\mathbf{u}_{2}-\mathbf{u}_{1}\right), \quad \tau_{s}=r_{s} \mathbf{u}_{1} .
$$

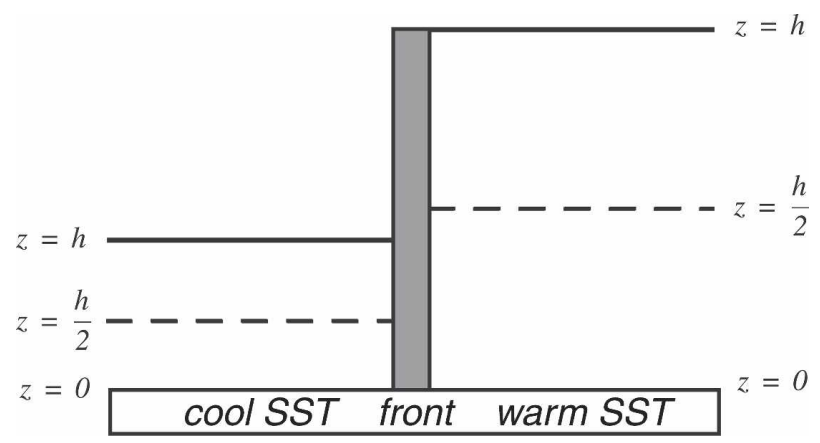

FIG. 2. Schematic of the model marine atmospheric boundary layer depth near an SST front. The depth $z=h$ is the level where the stress vanishes, which in the figure is deeper on the warm side of the SST front than on the cold side. The horizontal and vertical scales are several hundred kilometers and several hundred meters, respectively. With boundary layer depths as depicted, and with a fixed horizontal pressure gradient $G$ as in the text, the quasi-equilibrium near-surface winds will tend to be larger in the deep, convective boundary layer over the warm water than in the shallow, stable boundary layer over the cool water, because the vertical divergence of turbulent stress must remain constant as the layer depth $h$ increases, to balance the fixed pressure gradient. The division of the boundary layer into two sublayers considered in section $2 \mathrm{c}$ is indicated (dashed lines).

The effect of mean stability on turbulent vertical mixing will be modeled in the simplest possible way by specifying variations in the interfacial drag coefficient $r_{i}$ that reflects known qualitative behavior; stable conditions generally imply relatively weak mixing, and so can be represented by relatively small values of $r_{i}$, and unstable conditions by relatively large values of $r_{i}$. A more sophisticated one-dimensional quasi-equilibrium model, using any of a number of existing nonlinear, stability-dependent, turbulence closure schemes, could be formulated and solved numerically instead, but the present simplified approach is adequate to describe the conceptual issues and has the substantial advantage of yielding simple solutions in which the parameter dependence is clearly identifiable.

In the nonrotating $(f=0)$ case, the meridional momentum balance reduces to (2.2) when integrated over both layers, and the surface stress is independent of $r_{i}$. With rotation, solutions can conveniently be obtained numerically for the dimensionless velocities $\mathbf{u}_{j} / U_{g}$, where $U_{g}=G / f$ is the geostrophic velocity scale. The magnitude of the surface stress is $r_{s} U_{1}=r_{s}\left(u_{1}^{2}+v_{1}^{2}\right)^{1 / 2}$. For fixed $r_{s}, G$, and $f$, the surface stress is proportional to the dimensionless velocity $U_{1} / U_{g}$, and the ratio of the rotational time scale $1 / f$ to the internal frictional time scale $h /\left(2 r_{i}\right)$ is controlled entirely by variations in the internal turbulent mixing parameter $r_{i}$.

In the limits $r_{i} \rightarrow \infty$ and $r_{i} \rightarrow 0$, the two layer model is equivalent to the one-layer model with boundary 


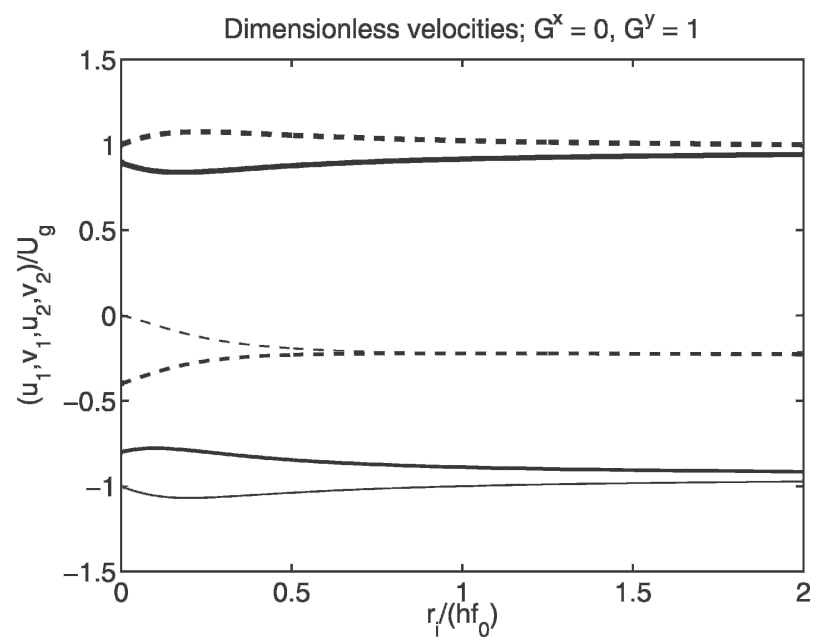

FIG. 3. Dimensionless magnitudes of lower-layer wind $U_{1} / U_{g}$ (thick solid line; $U_{1} / U_{g}>0$ ) and of upper-layer wind $U_{2} / U_{g}$ (thick dashed line; $\left.U_{2} / U_{g}>0\right)$ vs the dimensionless ratio $r_{i} /(h f)$ of rotational to frictional time scales. The zonal (solid) and meridional (dashed) wind components in the lower (thick lines) and upper (thin) layers of the two-layer model are also shown. The dimensionless pressure gradient is scaled to one and oriented meridionally $(G=1)$, so that the dimensionless wind components are all negative. For fixed pressure gradient $G$, drag coefficient $r_{s}$ boundary layer depth $h$, and Coriolis parameter $f$, the lower-layer wind speed $U_{1}$ and surface stress $r_{s} U_{1}$ depend only weakly on the internal mixing parameter $r_{i}$

layer depth $h$ or $h / 2$, respectively, and the wind speed and surface stress have the same one-layer dependence on boundary layer depth as discussed above. However, as the time-scale ratio based on the internal mixing parameter $r_{i}$ in the two-layer model varies over the range from 0 to 2 , the same range considered above for a similar time-scale parameter in the one-layer model, the dimensionless velocity $U_{1} / U_{g}$ and therefore also the surface stress vary by only about $10 \%$ (Fig. 3). This dependence of surface stress on the internal mixing is much weaker than the dependence of surface stress on boundary layer depth in the one-layer model (Fig. 1). The weak dependence of surface stress on the vertical distribution of turbulent mixing in the boundary layer in the rotating, two-layer model might be anticipated from the complete independence of these quantities in the nonrotating case that follows from the integral momentum balance (2.2).

\section{Discussion}

\section{a. Boundary layer depth coupling}

The quasi-equilibrium models presented above suggest a simple hypothesis as one possible contributing cause for the observed correlation of stress and SST: convective instability will lead to a deeper boundary layer over warmer water than over cooler water, and this will in turn lead to faster flow and higher stress over the warmer water, because the stress is distributed over a deeper layer. In the quasi-equilibrium balance, the mean stress divergence $\tau_{s} / h$ must balance the fixed pressure gradient force $G$. Thus, the stress $\tau_{s}$, and so also the low-level wind $v$ or $v_{1}$, will be systematically larger over warmer water, where $h$ is consistently larger, because the stress divergence is distributed over the deeper layer (Fig. 2). This is a robust mechanism, which is not qualitatively altered by the presence of rotation, and relies only on the well-known tendency of the stable boundary layer to be relatively shallow and the unstable boundary layer to be relatively deep, all other things being equal. This tendency derives from basic elements of the physics of stratified flow: the suppression of turbulence by stable stratification and the generation of turbulence by convective instability. Observationally, systematic boundary layer deepening over warm SST has been inferred, for example, from satellite observations of cloud liquid water (O'Neill et al. 2005), which show thicker low-level clouds over warmer water than over cooler water, and from direct measurements of the atmospheric boundary layer over SST fronts (Jury and Walker 1988; Jury 1993; Hashizume et al. 2002; Raymond et al. 2004). In the present quasi-equilibrium models, the horizontal changes in boundary layer depth are presumed to be confined to a small adjustment region around the SST front, and the adjusted boundary layer depth and surface stress are presumed to persist in a quasi-equilibrium state with small surface heat fluxes over the extended region of warm or cool water, resulting in a direct, positive, inphase correlation between surface stress and SST.

We hypothesize that this boundary layer depth effect is a major contributor to the extensive correlations of wind stress and SST on 50-500-km horizontal scales that have been discovered recently through the analyses of satellite remote sensing observations cited above in the introduction.

\section{b. Downward mixing of momentum}

As noted in the introduction, an alternate hypothesis for the observed correlations involves the control of downward momentum flux by the stability effect on the intensity of internal vertical mixing (e.g., Sweet et al. 1981; Wallace et al. 1989; de Szoeke and Bretherton 2004). It is useful to distinguish between the two different scenarios in which this mechanism may operate. The first is the quasi-equilibrium balance, represented in the simple models discussed above, in which the boundary layer dynamics are dominated by the stabil- 
ity-dependent stress divergence, the fixed pressure gradient, and the Coriolis force. The second explicitly involves the nonequilibrium effects associated with the transitions across SST fronts, and is discussed below in section 4. A related process that might also be considered a form of downward mixing involves the vertical flux of horizontal momentum associated with largescale subsidence. In this case, momentum is moved vertically by the subsidence and entrained into the momentum boundary layer. This process cannot be represented in the horizontally homogeneous, quasiequilibrium models discussed here, because mass conservation would require that the mean vertical velocity be balanced by a divergent horizontal flow. In principle, such a flow could contribute momentum to the boundary layer as an entrainment stress, which has been neglected here by assumption.

The analysis of the two-layer model shows that under the assumed quasi-equilibrium conditions, the surface stress $\tau_{s}=r_{s} U_{1}$ depends only weakly on the interfacial drag coefficient $r_{i}$, which represents internal turbulent mixing within the boundary layer. In the nonrotating limit, this result is independent of the assumed form of the stress law: the balance between surface stress and vertically integrated pressure gradient can be obtained directly from (2.2). The upper-layer flow $U_{2}=\left(u_{2}^{2}+v_{2}^{2}\right)^{1 / 2}$ does increase somewhat as $r_{i}$ decreases, implying that under stable conditions ( small $r_{i}$ ) there will be a jet in the upper part of the boundary layer, while under unstable conditions (large $r_{i}$ ) the vertical shear of the horizontal velocity in the boundary layer will be substantially reduced. This change in the vertical structure of the low-level winds is generally consistent with the available in situ atmospheric observations (e.g., Raymond et al. 2004; de Szoeke and Bretherton 2004). However, with $h$ and $G$ held constant, the stability effect on the intensity of internal turbulent mixing (i.e., on $r_{i}$ ) cannot substantially alter the near-surface winds or the wind stress. Thus, it is not possible in quasiequilibrium conditions to explain the strengthening of the near-surface winds and surface stress by invoking an increased downward flux of momentum resulting from the enhanced boundary layer turbulence that results from the passage of cool air over warm water.

\section{Nonequilibrium effects}

\section{a. Adjustment time scales}

The analytical models discussed above illustrate how under quasi-equilibrium conditions, and with fixed horizontal pressure gradients, correlations may naturally arise between boundary layer depth and surface stress. Whether these models are relevant to the obser- vations cited in the introduction and summarized by Xie (2004) and Chelton et al. (2004), which show correlated, in-phase variability of surface stress and SST over horizontal distances of 50-500 km, depends in part on the validity of the quasi-equilibrium hypothesis.

Two time scales are of principal interest. The first is the advective time scale $T_{A}=L / V$, where $V$ is a representative boundary layer wind speed, and $L$ is a characteristic horizontal scale for the transition region between cold and warm SST. The second is the surface drag time scale $T_{\tau}=h \Delta V / \tau$, where $h$ is the characteristic depth of the momentum boundary layer, $\Delta V$ is a measure of the change in wind speed across the transition region, and $\tau$ is a characteristic value of the surface stress. The time scale $T_{\tau}$ is an estimate of the time required for the surface drag $\tau$ to change the horizontal momentum of a boundary layer air column by the amount $h \Delta V$. Note that the advective time scale $T_{A}$ can also be interpreted as an entrainment time scale $T_{e}=$ $h / W_{e}$, for a characteristic entrainment velocity scale $W_{e}$ $=V h / L$ that is related to the wind speed by the boundary layer aspect ratio $h / L$. The ratio

$$
\gamma_{\tau A}=\frac{T_{\tau}}{T_{A}}=\frac{h \Delta V V}{\tau L}
$$

is a measure of the relative influence of the local surface stress and horizontal momentum advection on the flow in the boundary layer. When $\gamma_{\tau A}<1$, the local stress dominates, consistent with the quasi-equilibrium balance in the analytical models discussed above. When $\gamma_{\tau A}>1$, advection dominates, and the assumption of horizontal homogeneity in the quasi-equilibrium models is not justified. For the typical values $\Delta V=2.5$ $\mathrm{m} \mathrm{s}^{-1}, V=10 \mathrm{~m} \mathrm{~s}^{-1}$, and $\tau=0.1 \mathrm{~N} \mathrm{~m}^{-2}$, the condition $\gamma_{\tau A}<1$ is satisfied for horizontal scales $L>250 h$. For boundary layer depths $h=100-1000 \mathrm{~m}$, this corresponds to $L>25-250 \mathrm{~km}$, which is comparable to the horizontal scales of the observed variations. This supports the hypothesis that the processes described by the quasi-equilibrium models are relevant to the observations. The scaling also indicates that, with other parameters fixed, deeper boundary layers will tend to be more strongly controlled by lateral momentum advection, and shallower boundary layers more strongly controlled by surface stress.

\section{b. Downward mixing}

When $\gamma_{\tau A}>1$ and advection dominates, the nonequilibrium form of the downward-mixing hypothesis is appropriate for cold-to-warm flow. In this case, the advective balance implies conservation of the total horizontal momentum of a column of air that extends above 
the boundary layer over the upstream, cold SST but is fully entrained into the boundary layer over the downstream, warm SST. If, as is often the case, the flow in the free atmosphere just above the cool SST boundary layer is relatively fast, the subsequent vertical homogenization of horizontal momentum that is induced by convective instability over the warm SST will result in acceleration of the near-surface winds, and enhanced stress over the warm SST.

For warm-to-cold flow, however, the downwardmixing mechanism cannot operate in the opposite sense to reduce the stress over the cold downstream SST. To have this effect, it would have to separate the slowermoving air parcels in the deep, turbulent, upstream boundary layer from the faster-moving parcels, and preferentially shift these slower-moving parcels to the lower part of the boundary layer, creating a shallow downstream boundary layer with weaker winds, while preferentially detraining the faster-moving parcels out of the top of the boundary layer, creating faster, uncoupled flow above. Such an "upward unmixing" of momentum is an unphysical process that is inconsistent with accepted understanding of the mechanics of fluid turbulence. Thus, the downward-mixing mechanism cannot explain the downstream reduction of surface stress over a warm-to-cold SST transition. A cross section of such a warm-to-cold transition in the tropical Pacific, with a weakening of near-surface winds in a boundary layer of depth 300-500 m over the cold SST, is shown near $2^{\circ} \mathrm{N}, 124^{\circ} \mathrm{W}$, in Fig. 4 of Xie (2004).

\section{c. An LES simulation}

The observed correlations suggest a relatively symmetric wind stress response to cold-to-warm and warmto-cold SST transitions. The downward-mixing hypothesis cannot explain this symmetry, because, as described above, it does not predict the correct response either in the cold-to-warm transition or under quasiequilibrium conditions. In contrast, this symmetry is preserved by quasi-equilibrium models in which the wind stress is proportional to the local boundary layer depth. In the quasi-equilibrium models, which should provide useful guidance even in the vicinity of a transition if $\gamma_{\tau A}<1$, air-sea fluxes are presumed to cause the development of a shallow, stable boundary layer over the cold SST. Because the surface stress $\tau$ is then confined to a shallow layer with small depth $h$, the magnitude of the stress divergence $\tau / h$ will increase, and the near-surface flow can be expected to decelerate proportionally. Thus, the simple models suggest that boundary layer depth coupling can operate to reduce the stress over the cold downstream SST in warm-tocold flow.
To explore this effect in a model that can resolve the transition region, a limited set of numerical integrations were carried out using a three-dimensional, rotating, nonhydrostatic, turbulence-resolving, large eddy simulation (LES) numerical model. The model (Skyllingstad 2003; Skillingstad et al. 2005) is based on the equations of motion given in Deardorff (1980), with the addition of an open boundary condition on the downwind boundary allowing for changes in the boundary layer structure. The domain contained an upstream region with uniform SST, a transition region with an SST gradient, and a downstream region with uniform SST that differed from the upstream SST. The model was initialized with an 80-m-deep mixed layer capped with an inversion and was forced with a constant geostrophic wind of $6 \mathrm{~m} \mathrm{~s}^{-1}$. The prescribed upstream SST was $2^{\circ} \mathrm{C}$ warmer than the initial boundary layer temperature, and the upstream $(0<y<4 \mathrm{~km})$ flow was allowed to evolve to a quasi-equilibrium structure using a recirculating configuration with an internal periodic boundary following Mayor et al. (2002). An abrupt $4^{\circ} \mathrm{C}$ transition to colder downstream SST was specified over the region $4<y<5 \mathrm{~km}$. Uniform cold SST was prescribed from the downstream edge of the transition at $y=5 \mathrm{~km}$ to the outflow boundary at $y=19 \mathrm{~km}$. The vertical and horizontal grid resolution was $4 \mathrm{~m}$. The spatial scales and SST gradient strength were chosen for computational convenience, and differ significantly from those of the observations discussed above. However, this difference does not affect the central point of the simulation, which is to demonstrate that the warm-to-cold transition can be controlled by the surface stress and boundary layer depth changes, but not by upward unmixing. A more comprehensive analysis of these LES simulations, including similar cases with cold-to-warm flow, is in progress and will be reported elsewhere.

In the warm-to-cold simulation, a deep boundary layer develops over the warm upstream SST, with relatively strong $\left(5 \mathrm{~m} \mathrm{~s}^{-1}\right)$ winds (Fig. 4). The vertical profile of turbulent stress $\left\langle v^{\prime} w^{\prime}\right\rangle$, where the perturbation velocities $v^{\prime}$ and $w^{\prime}$ are computed relative to a local cross-stream mean and the angle brackets indicate an average over $0<y<4 \mathrm{~km}$, shows that the momentum boundary layer extends to over $150 \mathrm{~m}$ over the warm upstream SST (Fig. 5). In the downstream region, a shallow boundary layer with weak near-surface winds develops over the cold SST, with stronger winds above (Figs. 4, 6). The corresponding profiles of turbulent stress show a shallow momentum boundary layer over the cold downstream SST, consistent with the nearsurface wind profile and the downstream acceleration above the boundary layer (Fig. 5). This structure is consistent with the assumption that the downstream dy- 

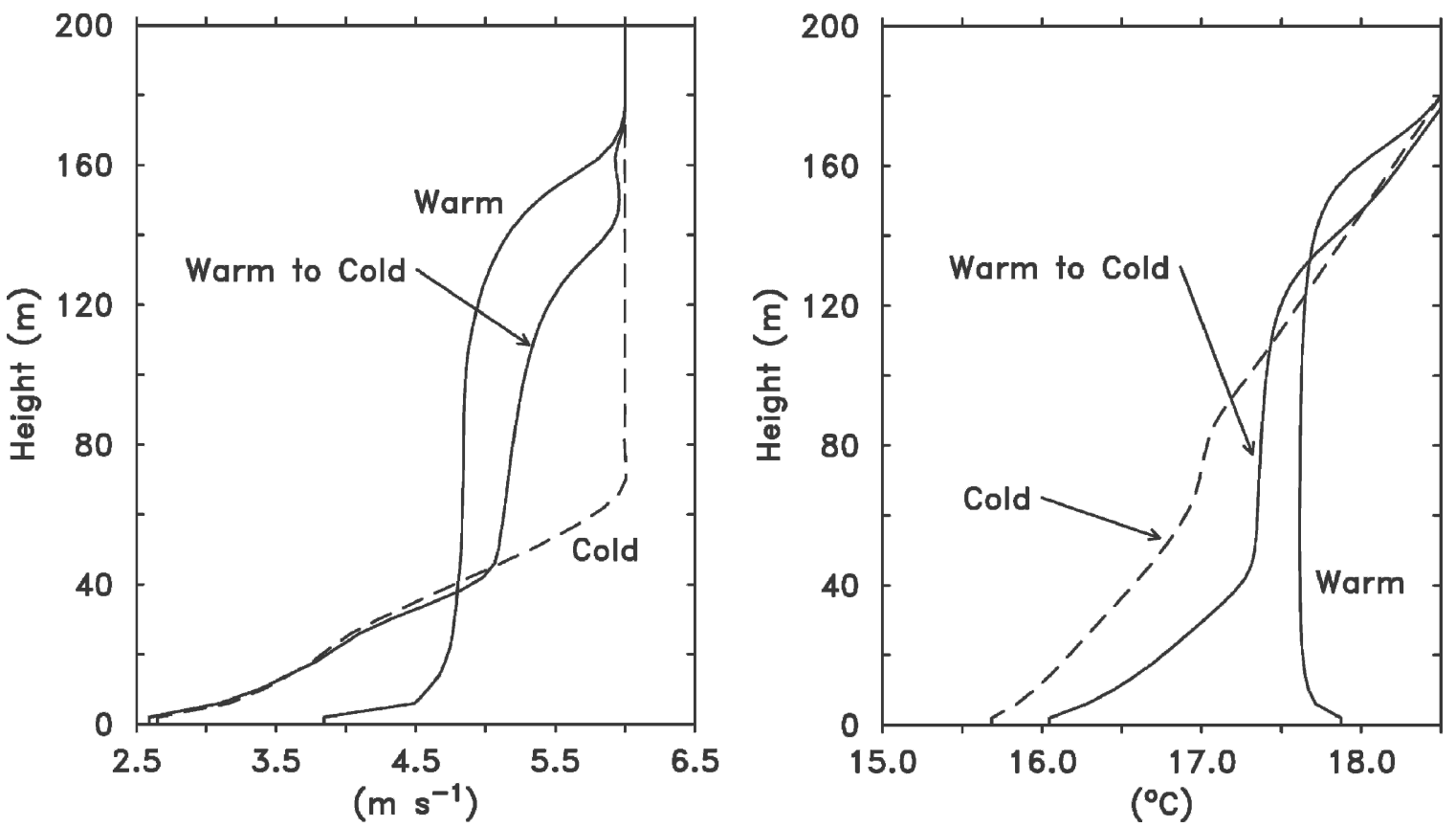

FIG. 4. LES model meridional (left) wind and (right) temperature vs height (m) over the warm SST in the upstream region (solid line, "Warm") and the cold SST in the downstream region (solid, "Warm to Cold"). The profiles over the horizontally uniform cold SST are also shown (dashed, "Cold").

namics are dominated by the surface stress acting on the shallow, stable, downstream boundary layer, which efficiently decelerates the near-surface flow. With $h=$ $20 \mathrm{~m}, \Delta V=2 \mathrm{~m} \mathrm{~s}^{-1}, V=4 \mathrm{~m} \mathrm{~s}^{-1}, \tau=0.02 \mathrm{~N} \mathrm{~m}^{-2}$, and $L=15 \mathrm{~km}$, the time-scale ratio $\gamma_{\tau A} \approx 0.5<1$, indicating that the surface stress should indeed control the downstream momentum balance. As noted above, the downward-mixing hypothesis can only explain this deceleration by invoking an unphysical upward unmixing of momentum.

To examine the dependence of the downstream state on horizontal gradients, a second simulation was conducted with horizontally homogeneous conditions and with SST that was temporally varied from an initially neutral (i.e., SST equal to boundary layer air temperature) upstream value to the cold downstream value. The shallow boundary layer structure and near-surface wind and stress profiles that developed in this "cold" case were very similar to those that developed over the cold downstream SST in the warm-to-cold transition case (Figs. 4, 5). Indeed, the wind profiles in the two stable boundary layers are nearly identical below $40 \mathrm{~m}$. This illustrates that the role of horizontal gradients, including both momentum advection and thermodynamically induced pressure gradients, in setting the downstream, shallow boundary layer and wind structure is small. Direct evaluation of the momentum balances also confirms that the horizontal pressure gradi-

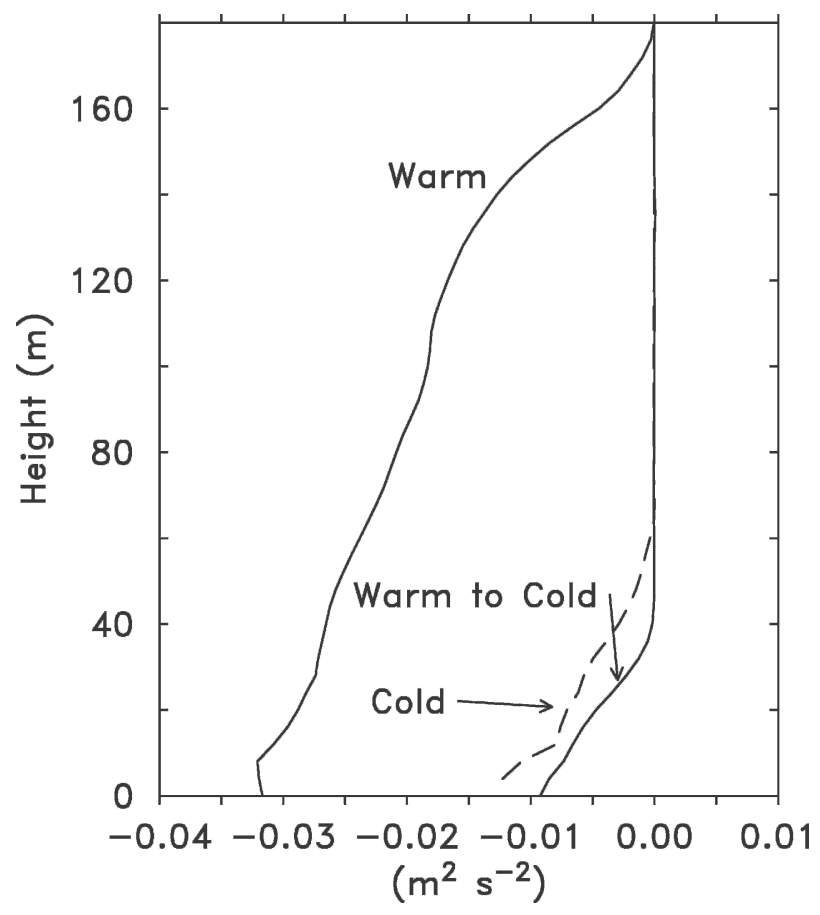

FIG. 5. LES model turbulent vertical flux $\left\langle v^{\prime} w^{\prime}\right\rangle$ of horizontal momentum averaged over the upstream $(0<x<4 \mathrm{~km}$; solid line, "Warm") and downstream $(15<y<19 \mathrm{~km}$; solid, "Warm to Cold") regions vs height $(\mathrm{m})$. This includes the stress that is carried by the subgrid-scale parameterization, which is the dominant contribution at the lowest few grid points but is negligible elsewhere. The profile over the horizontally uniform cold SST is also shown (dashed line, "Cold"). 


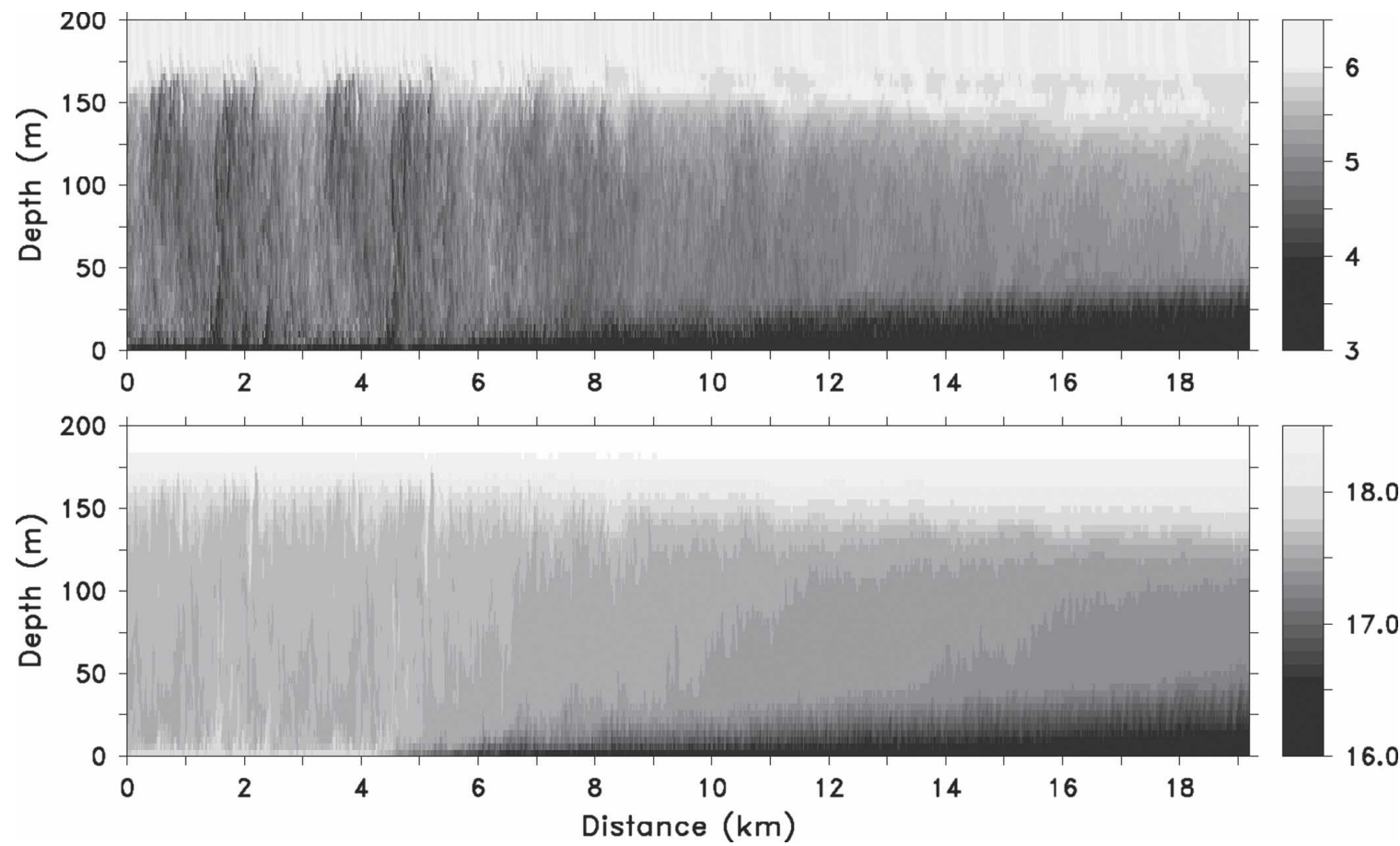

FIG. 6. LES model meridional (top) wind and (bottom) temperature vs meridional distance ( $\mathrm{km}$ ) and height (m). The upstream $(0<y<4 \mathrm{~km}) \mathrm{SST}$ is $2^{\circ} \mathrm{C}$ warmer and the downstream $(5<y<20 \mathrm{~km}) \mathrm{SST} 2^{\circ} \mathrm{C}$ colder than the initial boundary layer temperature.

ents are small in the warm-to-cold simulation. However, it should also be noted that, because the time scales of these simulations are shorter than the inertial time scale $1 / f$, where $f$ is the Coriolis parameter, the momentum balance remains time dependent in these simulations, in contrast to the steady balance in the quasi-equilibrium analytical models, with the primary balance between the stress divergence and the local and (in the warm-to-cold case) advective rates of change of momentum. Thus, while the structure of the stress divergence and the near-surface winds and temperature are generally consistent with that in the analytical models, the full momentum balances in these simulations are more complex.

In general, many factors may control the extent of the transition region around an SST front over which the quasi-equilibrium assumption is not appropriate. The simple, quasi-equlibrium models presented in section 2 neither address the force balances in the transition region nor yield any information about the spatial or temporal rates at which equilibrium is established, beyond the simple scaling arguments discussed in section $4 \mathrm{a}$. Note that the rate at which the boundary layer depth changes because of entrainment (e.g., Stevens et al. 2002) or the rate at which the boundary layer temperature changes because of surface fluxes and entrainment (Small et al. 2005) may also play a role in setting the equilibrium time scale for the wind stress. In addition, thermodynamically induced pressure gradients and other effects may be relatively larger in many of the observed open-ocean regions, which involve larger scales and deeper boundary layers than are represented in the LES simulations. The present LES simulations serve primarily to illustrate that the near-surface deceleration in the warm-to-cold transition cannot be explained in terms of downward momentum transport, and to demonstrate that there are geophysically relevant conditions under which the quasi-equilibrium balance can provide useful insight into the correlations of SST and stress associated with the warm-to-cold transition. They do not, and are not intended to, provide a definitive demonstration that excludes all other possible causes of the observed correlations.

\section{Conclusions}

Simple quasi-equilibrium models are used to explore hypotheses related to observed spatial correlations, on horizontal scales of $50-500 \mathrm{~km}$, between sea surface temperature and wind stress. It is argued that changes in stress boundary layer depth are plausible causes of the observed correlations, but that, under quasiequilibrium conditions in which a one-dimensional bal- 
ance prevails, a hypothesized stability effect on the downward mixing of momentum cannot explain the observations. One version of the downward-mixing hypothesis is appropriate under certain circumstances for the cold-to-warm SST transition, but would have to be supplemented by an unphysical "upward unmixing" hypothesis in order to explain the deceleration of the near-surface flow in warm-to-cold SST transitions. Idealized LES model simulations show an evolution of the stress profiles that is consistent with that of the theory. A dimensionless ratio $\gamma_{\tau A}$ is introduced to measure the relative influence of lateral momentum advection and local surface stress on the boundary layer wind profile. It is argued that when $\gamma_{\tau A}<1$, the boundary layer depth effect will dominate lateral advection and control the response.

The analytical model considered here cannot address the physics of the atmospheric boundary layer in the immediate vicinity of an SST front. Other potentially important mechanisms not explored here include thermodynamically induced pressure gradients (e.g., Lindzen and Nigam 1987; Wai and Stage 1989; Small et al. 2003). However, the present model can account for the observed influence of SST on low-level winds in the far field, where quasi-equilibrium conditions are more likely to pertain; the model predicts that, for a given fixed pressure gradient, the low-level winds will be stronger over warmer water than over cooler water, because of the greater depth of the stress boundary layer over the warmer water. Future work should evaluate the role of this mechanism in more realistic simulations and through the analysis of direct observations of the response of marine atmospheric boundary layer structure and dynamics to changes in SST. It may also be possible to develop useful, idealized dynamical coupled ocean-atmosphere models of this effect by extending parameterizations such as (2.2) or (2.7) to include a dependence of atmospheric boundary layer depth on local SST.

A related question involves the influence of the observed boundary layer coupling on the free atmosphere. In this context, it is interesting to note a simple extension of the Ekman theory (2.7) discussed above. Let the interior geostrophic flow in the free atmosphere above the boundary layer be $\left(U_{G}, V_{G}\right)$, and assume that the Ekman depth $\delta$ is correlated locally with an SST field that varies slowly in the horizontal. Then, the Ekman vertical velocity $W_{E}$ induced by the corresponding Ekman transport divergence is

$$
W_{\infty}=\frac{1}{\sqrt{2}} \mathbf{U}_{\delta} \cdot \nabla \delta
$$

where

$$
\mathbf{U}_{\delta}=\frac{1}{\sqrt{2}}\left(U_{G}-V_{G}, V_{G}+U_{G}\right)
$$

is a velocity with the magnitude of the interior geostrophic flow and rotated $45^{\circ}$ counterclockwise from the interior flow. This is the same as the vertical velocity that would be induced, under the usual linearized kinematic surface boundary condition, by a flow $\mathbf{U}_{\delta}$ over orography of amplitude $\delta / \sqrt{2}$. Other effects, including radiative and moist processes, may lead to additional coupling between the boundary layer and the free atmosphere.

Acknowledgments. The work was supported by the Office of Naval Research, under Grants N00014-98-10813 and N00014-01-1-0231; the National Science Foundation, under Grants OCE-9907854, OCE-0218812, and ATM-0002322; and the National Aeronautics and Space Administration, under Grant NAS5-32965. We are grateful for the comments of three anonymous reviewers, which led to improvements in the presentation.

\section{REFERENCES}

Chelton, D. B., and Coauthors, 2001: Observations of coupling between surface wind stress and sea surface temperature in the eastern tropical Pacific. J. Climate, 14, 1479-1498.

_ - M. G. Schlax, M. H. Freilich, and R. F. Milliff, 2004: Satellite measurements reveal persistent small-scale features in ocean winds. Science, 303, 978-983.

Deardorff, J. W., 1980: Stratocumulus-capped mixed layers derived from a three-dimensional model. Bound.-Layer Meteor., 18, 495-527.

de Szoeke, S., and C. Bretherton, 2004: Quasi-Lagrangian large eddy simulations of cross-equatorial flow in the East Pacific atmospheric boundary layer. J. Atmos. Sci., 61, 1837-1858.

Hashizume, H., S.-P. Xie, W. T. Liu, and K. Takeuchi, 2001: Local and remote atmospheric response to tropical instability waves: A global view from space. J. Geophys. Res., 106, $10173-10185$.

,$- \ldots$, M. Fujiwara, M. Shiotani, T. Watanabe, Y. Tanimoto, W. T. Liu, and K. Takeuchi, 2002: Direct observations of atmospheric boundary layer response to slow SST variations over the eastern equatorial Pacific. J. Climate, 15, 3379-3393.

Jury, M. R., 1993: A thermal front within the marine atmospheric boundary layer over the Agulhas Current south of Africa: Composite aircraft observations. J. Geophys. Res., 99, 32973304.

— , and N. Walker, 1988: Marine boundary layer modification across the edge of the Agulhas Current. J. Geophys. Res., 93, 647-654.

Lindzen, R., and S. Nigam, 1987: On the role of sea surface temperature gradients in forcing low-level winds and convergence in the Tropics. J. Atmos. Sci., 44, 2418-2436.

Liu, W. T., X. Xie, P. Polito, S.-P. Xie, and H. Hashizume, 2000: Atmospheric manifestation of tropical instability waves observed by QuikSCAT and Tropical Rain Measuring Mission. Geophys. Res. Lett., 27, 2545-2548. 
Mayor, S. D., P. R. Spalart, and G. J. Tripoli, 2002: Application of a perturbation recycling method in the large-eddy simulation of a mesoscale convective internal boundary layer. J. Atmos. Sci., 59, 2385-2395.

O'Neill, L., D. Chelton, and S. Esbensen, 2003: Observations of SST-induced perturbations of the wind stress field over the Southern Ocean on seasonal timescales. J. Climate, 16, 2340 2354.

- — — - — , and F. Wentz, 2005: High-resolution satellite measurements of the atmospheric boundary layer response to SST perturbations over the Agulhas Return Current. J. Climate, 18, 2706-2723.

Pedlosky, J., 1987: Geophysical Fluid Dynamics. 2d ed. Springer, $710 \mathrm{pp}$.

Polito, P., J. Ryan, T. Liu, and F. Chavez, 2001: Oceanic and atmospheric anomalies of tropical instability waves. Geophys. Res. Lett., 28, 2233-2237.

Raymond, D. J., and Coauthors, 2004: EPIC2001 and the coupled ocean-atmosphere system of the tropical East Pacific. Bull. Amer. Meteor. Soc., 85, 1341-1354.

Skyllingstad, E. D., 2003: Large-eddy simulation of katabatic flows. Bound.-Layer Meteor., 106, 217-243.

_ R. M. Samelson, L. Mahrt, and P. Barbour, 2005: A numeri- cal modeling study of warm offshore flow over cool water. Mon. Wea. Rev., 133, 345-361.

Small, R. J., S.-P. Xie, and Y. Wang, 2003: Numerical simulation of atmospheric response to Pacific tropical instability waves. J. Climate, 16, 3723-3741.

S. K. Esbensen, and D. Vickers, 2005: Numerical simulation of boundary layer structure and cross-equatorial flow in the Eastern Pacific. J. Atmos. Sci., 62, 1812-1830.

Stevens, B., J. Duan, J. McWilliams, M. Münnich, and J. D. Neelin, 2002: Entrainment, Rayleigh friction, and boundary layer winds over the tropical Pacific. J. Climate, 15, 30-44.

Sweet, W., R. Fett, J. Kerling, and P. LaViolette, 1981: Air-sea interaction effects in the lower troposphere across the north wall of the Gulf Stream. Mon. Wea. Rev., 109, 1042-1052.

Wai, M., and S. Stage, 1989: Dynamical analyses of marine atmospheric boundary layer structure near the Gulf Stream oceanic front. Quart. J. Roy. Meteor. Soc., 115, 29-44.

Wallace, J., T. Mitchell, and C. Deser, 1989: The influence of sea-surface temperature on surface wind in the eastern equatorial Pacific: Seasonal and interannual variability. J. Climate, 2, 1492-1499.

Xie, S.-P., 2004: Satellite observations of cool ocean-atmosphere interaction. Bull. Amer. Meteor. Soc., 85, 195-208. 